\title{
How Stochastic Network Calculus Concepts Help Green the Power Grid
}

\author{
Kai Wang*†, Steven Low ${ }^{\dagger}$ and Chuang Lin* \\ *Dept. of Computer Science and Technology, Tsinghua University, China \\ ${ }^{\dagger}$ Computing and Mathematical Science, California Institute of Technology, USA \\ \{kaiphd, slow\}@caltech.edu, chlin@tsinghua.edu.cn
}

\begin{abstract}
The renewable energy generation such as solar and wind will constitute an important part of the next generation grid. As the variations of renewable sources may not match the time distribution of load, energy storage is essential for grid stability. Supplemented with energy storage, we investigate the feasibility of integrating solar photovoltaic (PV) panels and wind turbines into the grid. To deal with the fluctuation in both the power generation and demand, we borrow the ideas from stochastic network calculus and build a stochastic model for the power supply reliability with different renewable energy configurations. To illustrate the validity of the model, we conduct a case study for the integration of renewable energy sources into the power system of an island off the coast of Southern California. Performance of the hybrid system under study is assessed by employing the stochastic model, e.g., with a set of system configurations, the long-term expected Fraction of Time that energy Not-Served (FTNS) of a given period can be obtained.
\end{abstract}

\section{INTRODUCTION}

The need to reduce greenhouse gas emissions is moving its attention to more environmentally-friendly and sustainable energy sources. Alternative energy resources such as solar and wind has greatly increased investment due to their environmental friendliness and decreasing prices of turbines and photovoltaic (PV) panels. There is an emerging trend that the next-generation grid will feature renewable-energy sources to reduce the carbon footprint of the grid, making it greener. However, renewable energy sources tend to be highly variable: solar energy goes to the grid during the day, wind can pick up power at night. The drawback, common to solar and wind options, is their unpredictable nature and dependence on weather and climatic changes, and the variations of solar and wind energy may not match with the time distribution of load demand.

The integration of renewable energy resources into the electric grid presents challenges due to the intermittency in the power generation. These difficulties can be alleviated by effectively utilizing intermediate energy storage, such as batteries to improve power reliability and balance the integration of renewable wind and solar energy into the large generation portfolio. Advanced storage technology plays an important role in smoothing the gap between supply and demand. It is recently reported that the Los Angeles Department of Water and Power (LADWP) has formed a partnership with BYD Ltd. Corp. on a grid-scale battery project for renewable energy storage, which will lead to the development of a power storage unit up to $10 \mathrm{MWh}$ [13]. It is quite promising that the grid would incorporate large power storage unit in the near future.

Prior to construction of a renewable generation station, it is necessary to determine the optimum number of PV panels and wind turbines to continuously match generated energy and desired consumption, although both can change dramatically over time period of a few hour. Aggregating inherently stochastic power sources to achieve reliable electricity supply is a non-trivial problem. Various optimization techniques for hybrid PV/wind systems sizing have been proposed in the literature [12], such as probabilistic approach [10] [14], graphical construction technique [9], artificial intelligence methods [11], and iterative technique [15].

By careful examination of the Internet and the grid, we show how similar Internet concepts can be used to address the above problem in the development of a green grid, as both the Internet and the electrical grid are designed to provider some services, i.e., for information and energy respectively. The provision of stochastic service guarantees motivates us to use stochastic network calculus, and we initiate a dialogue between these two communities. Network calculus [8] is a theory that uses bounds to deal with queuing systems in computer networks, whose focus is on performance guarantees. Its essential idea is to use alternative algebra to transform a complex non-linear system into an analytically tractable linear system.

However, solutions to problems in the communication network research could not be directly applicable to the grid. We define the energy supply curve in the power grid, which is different from service curve in network calculus whose definition is coupled with the arrival curve [6][7][8]. In addition, in stochastic network calculus [1], by assuming a lossless system, results obtained from the backlog analysis are used directly to approximately describe the loss, which leads to loose bounds. Based on the virtual queueing system in the power network, we derive a non-recursive formula for the buffer overflow probability, and further derive the performance metrics in concern. By adjusting and modifying the framework of stochastic network calculus, we then build a stochastic model to analyze the achievable level of performance with given system configurations. To illustrate the validity of the model, we conduct a case study for the integration of renewable-energy sources into the power system of an island off the coast of Southern California. Performance 


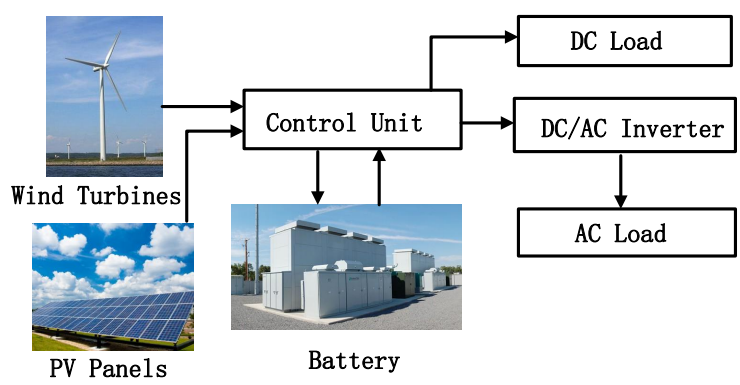

Fig. 1. Schematic of the hybrid power system

of the hybrid system under study is assessed by employing the stochastic model, e.g., with a set of system configurations, the long-term expected Fraction of Time that energy is Not-Served (FTNS) of a given period can be obtained.

The paper is organized as follows. We first present the integrated system considered in this paper in Section 2. Borrowing the ideas from stochastic network calculus, we define the energy demand and supply processes, and then build a stochastic model for the power system. In Section 3, the performance metrics of interest are derived. In the following section, we conduct a case study for the integration of renewable-energy sources into the power system of an island. Performance of the hybrid system under study is assessed by employing the stochastic model. Finally, we summarize the contributions of this paper and conclude in Section 5.

\section{Power System Modeling}

\section{A. Problem Description}

We consider a scenario where the electric network is supplied by a combination of solar PV panels, wind turbines and battery. A hybrid system consists of solar PV, wind turbine, battery, and controller etc., as shown in Fig. 1. The PV panels and wind turbines work together to satisfy the load demand. When the energy sources are abundant, the excess power generation will feed the battery until it is fully charged. Whenever there is a deficiency in power, the battery will be discharged to cover the load requirements until the energy storage is depleted.

In the face of fluctuations in both power load and generation, we try to investigate the effects of energy storage on the power supply reliability in configurations with different levels of renewable generation. We are interested in quantifying the Loss of Power Supply Probability (LPSP) at a given time, as well as the long-term expected Fraction of Time energy Not-Served (FTNS) during a time period. In general, the LPSP/FTNS is a function of the total area $N_{p}$ of PV panels, the number $N_{w}$ of wind turbines, and the specified capacity $C$ of battery storage (other factors are ignored here). They are design-time decisions to be made before investing in the deployment of renewable energy sources and energy storage.

\section{B. Formulation and Notations}

The hybrid solar-wind system design depends on the performance of individual components. In order to predict the sys- tem's performance, individual components should be modeled and then the reliability of the overall system can be evaluated. The stochastic nature of the load and renewable generation feature fluctuations motivates us to build a system model based on similar concepts in stochastic network calculus [1][6].

Consider a power system where the time axis $T$ is discretized into 1 hour. In time interval $[0, t],(t \leq T)$, we denote by $D(t)$ the cumulative amount of energy demand in the system (in MWh); by $S(t)$ the cumulative amount of energy supply in the system. We call $D(t)$ the energy demand process, and $S(t)$ the energy supply process of the system, with $D(0)=S(0)=0$. For any $0 \leq s \leq t$, we denote $D(s, t)=D(t)-D(s)$, and $S(s, t)=S(t)-S(s)$.

In this paper, we use $[x]^{+}$to denote the maximum of a given number of $x$ and 0 , i.e., $[x]^{+} \equiv \max \{x, 0\}$. The Minplus Convolution of functions $a(x)$ and $b(x)$, denoted by $\otimes$, is defined as follows:

$$
(a \otimes b)(x)=\inf _{0 \leq y \leq x}[a(y)+b(x-y)] .
$$

\section{Modeling of Energy Storage}

Depending on the PV and wind energy production and the energy demand, the state $b(t)$ of battery at time $t$ can be calculated as follows: If the energy generated from the $\mathrm{PV} /$ wind system is greater than the load for a particular hour, the energy surplus is stored in the battery and the battery is charged:

$$
b(t)=\min \left[C, b(t-1)+[S(t-1, t)-D(t-1, t)] \eta_{c}\right],
$$

where $\eta_{c}$ denotes the charge efficiency of the battery. When the battery reaches its maximum value, which is determined by the specified battery capacity $C$, any excess energy generated cannot be charged and is wasted.

When the energy demand is greater than the supply for a particular hour, the battery will be discharged to supplement the supply. In this case, the new state of the battery is:

$$
b(t)=\left[b(t-1)-[D(t-1, t)-S(t-1, t)] \eta_{d}\right]^{+},
$$

where $\eta_{d}$ denotes the discharge efficiency of the battery. Due to the physical constraints, the minimal quantity level of battery is determined by the maximum depth of discharge. If the battery decreases to its minimum value $C_{0}$, the deficient energy demand cannot be meted out from the battery system, and we call this loss of power supply at time $t$. In this paper, we suppose $\eta_{c}=\eta_{d}=1$ and $C_{0}=0$.

\section{Modeling of Energy Demand}

In order to guarantee a certain level of performance, the energy demand must be limited. Intuitively, a deterministic demand curve implies that the amount of energy demand in any time interval $(s, t]$ is upper-bounded by some function $\alpha(t-s)$. By introducing a violation probability, we can find its stochastic counter-part. Throughout this paper, we assume all demand curve, supply curve functions are non-negative and non-decreasing, and all bounding functions are non-negative and non-increasing. 
Definition 1: A energy demand process $D(t)$ is said to have a stochastic demand curve $\alpha(t)$ with bounding function $\varepsilon_{d}$, denoted by $D \sim\left\langle\varepsilon_{d}, \alpha\right\rangle$, if for all $t \geq 0$ and all $x \geq 0$, there holds

$$
\mathbb{P}\left\{\sup _{0 \leq s \leq t}[D(s, t)-\alpha(t-s)]>x\right\} \leq \varepsilon_{d}(x) .
$$

\section{E. Modeling of Energy Supply}

The supply curve defines a lower bound on the energy provided by the power generator. Also by introducing a violation probability, we can make sure that the power supply process is above some lower bound with a high probability.

Definition 2: A energy supply process $S(t)$ is said to provide a stochastic supply curve $\beta(t)$ with bounding function $\varepsilon_{s}$, denoted by $S \sim\left\langle\varepsilon_{s}, \beta\right\rangle$, if for all $t \geq 0$ and all $x \geq 0$, there holds

$$
\mathbb{P}\left\{\sup _{0 \leq s \leq t}[\beta(t-s)-S(s, t)]>x\right\} \leq \varepsilon_{s}(x) .
$$

\section{F. A Word on Model Tightness}

Generally speaking, there is a tradeoff between the energy demand curve and its bounding function. We can tune the demand curve by relaxing the function $\alpha(t)$. These relaxations are critical to the derivation of a tight bounding function, and we can get series of energy demand curves. We should neither select a very loose demand curve to make the bounding function small, nor use a very tight demand curve such that the bounding function becomes meaningless. Based on an existing one with a given acceptable range on the bounding function, we can search for a tighter demand curve within the given tolerance bound as long as it exists.

Similarly, when we select a energy supply curve, we need to make the tradeoff between the tightness of the supply curve and the usefulness of the bounding function. Given a supply curve and the tolerance bound for the bounding function, we can also search for a tighter supply curve in the same way to the demand curve.

\section{System REliability ANALYSis}

However, the concepts of stochastic network calculus could not be applied directly to the power network. In network calculus, the service characterization is linked to the traffic arrival [1][8]. In power system, however, the definition of energy generation and load are decoupled. In addition, with the definition of backlog in network calculus, it could only be derived if both the arrival process and departure process of a queue were known [1][6][7][8]. In the virtual queueing system below, there is no such "energy departure process" in correspondence to the energy demand process. We have to adjust the theory framework to analyze the power system.

\section{A. Queueing Principles of the Power System}

We then analogize the energy demand and supply to a virtual queueing system. Without loss of generality, the battery is fully charged initially. Regarding the power demand as the arrival of the queue, the energy supply as the service provided by the queue, then the buffering process of the virtual queue could be considered as the discharging process of the battery. We denote the buffer for the virtual queueing system at time $t$ as $B(t)$, which can be considered as the deficit energy storage of the system, and we have $B(t)=C-b(t)$.

When the energy generated is greater than the energy demand in a certain period of time, as long as the maximum battery capacity is not reached, the buffer decreases which means that it is charged. When the load is greater than the energy supply in a time period, the buffer increases, i.e., the battery is discharged until it decreases to the minimum level. Given the actual storage capacity at time $t, C(t)$, we have:

$$
B(t)=\min \left[C(t),[B(t-1)+D(t-1, t)-S(t-1, t)]^{+}\right],
$$

where $C(t)$ may not be equal to the specified storage capacity $C$, i.e., it might varies as physical/environmental conditions change. We assume that the actual storage capacity $C(t)=C$, for $t>0$, and $C(0)=0$. As assumed above, the battery is fully charged in the beginning, i.e., $B(0)=0$. We can derive a non-recursive identity for the virtual buffer size:

$B(t)=\inf _{0 \leq s \leq t}\left[\sup _{s \leq u \leq t}[D(u, t)-S(u, t), D(s, t)-S(s, t)+C(s)]\right]$.

Due to space limitations, here we only show a sketch of the proof for (7). We can first prove $B(t) \leq \sup _{s \leq u \leq t}[D(u, t)-$ $S(u, t), D(s, t)-S(s, t)+B(s)]$ by induction. With $B(t) \leq$ $C(t)$, we could get $B(t) \leq \inf _{0 \leq s \leq t}\left[\sup _{s \leq u \leq t}[D(u, t)-\right.$ $S(u, t), D(s, t)-S(s, t)+C(s)]]$. Also with the assumption, we have $B(0)=C(0)=0$, and there must be some $s=\sup \{v \mid B(v)=C(v), 0 \leq v \leq t\}$. The proof is completed as there exits an $s, 0 \leq s \leq t$, such that $B(t)=\sup _{s \leq u \leq t}[D(u, t)-S(u, t), D(s, t)-S(s, t)+C(s)]$.

\section{B. Performance Metrics}

For a specified energy storage capacity $C$, we are interested in the probability that the energy demand cannot be met by the generation and the stored energy in the battery. In time slot $t$, $(t \geq 1)$, if $[B(t-1)+D(t-1, t)-S(t-1, t)]^{+}>C$, we call this Loss of Power Supply (LPS) at time $t$, which is dependent on the storage capacity $C$, which satisfies $L P S(C, t)=[B(t-$ 1) $+D(t-1, t)-S(t-1, t)-C]^{+}$. Applying (7),

$$
\begin{aligned}
L P S(C, t)= & \inf _{0 \leq s \leq t-1}\left[\sup _{s \leq u \leq t-1}[D(u, t)-S(u, t)-C,\right. \\
& \left.D(s, t)-S(s, t)+C(s)-C]^{+}\right]
\end{aligned}
$$

With the battery storage capacity $C$ (in MWh), the Loss of Power Supply Probability $\operatorname{LPSP}(C, t)$ at time $t$ equals to $\mathbb{P}\{L P S(C, t)>0\}$. The long-term expected Fraction of Time energy Not-Served (FTNS) over the period $[0, T]$, $\operatorname{FTNS}(C, T)$, is given by:

$$
\operatorname{FTNS}(C, T)=\frac{1}{T} \Sigma_{t=1}^{T} \operatorname{LPSP}(C, t) .
$$

Give a storage capacity $C$, we introduce the following theorem to calculate the $\operatorname{LPSP}(C, t)$. 
Theorem 1: (Loss of Power Supply Probability) For a virtual queue in the power network, if the energy demand process has a stochastic demand curve $\alpha$ with bounding function $\varepsilon_{d}$, i.e., $D \sim\left\langle\varepsilon_{d}, \alpha\right\rangle$, and the system provides a stochastic supply curve $\beta$ with bounding function $\varepsilon_{s}$, i.e., $S \sim\left\langle\varepsilon_{s}, \beta\right\rangle$, then for all $t \geq 0$ and all $x \geq 0$, given storage capacity $C, \operatorname{LPSP}(C, t)$ can be calculated by:

$$
\begin{aligned}
\operatorname{LPSP}(C, t) & =\mathbb{P}\{L P S(C, t)>0\} \\
& \leq \varepsilon_{d} \otimes \varepsilon_{s}\left(C-\sup _{0 \leq s \leq t}[\alpha(s)-\beta(s)]\right) .
\end{aligned}
$$

To prove Theorem 1, we first introduce a lemma as follows, which based on the min-plus convolution [1].

Lemma 1: For any random variables $X$ and $Y$, with their complementary cumulative distribution functions (CCDF) $F^{c}(x)$ and $G^{c}(x)$. No matter whether they are independent or not, if $F^{c}(x) \leq \varepsilon_{f}(x)$ and $G^{c}(x) \leq \varepsilon_{g}(x)$, there holds,

$$
\mathbb{P}\{X+Y>x\} \leq F^{c}(x) \otimes G^{c}(x) \leq \varepsilon_{X} \otimes \varepsilon_{Y}(x)
$$

With Lemma 1, we then prove Theorem 1 as follows:

Proof: From (8), we have that for $t \geq 1$,

$$
\begin{aligned}
& \mathbb{P}\{L P S(C, t)>0\}=\mathbb{P}\left\{\operatorname { i n f } _ { 0 \leq s \leq t - 1 } \left[\sup _{s \leq u \leq t-1}[D(u, t)\right.\right. \\
& -S(u, t)-C, D(s, t)-S(s, t)+C(s)-C]]>0\} .
\end{aligned}
$$

For the right hand side, we have:

$$
\begin{aligned}
& \inf _{0 \leq s \leq t-1}\left[\sup _{s \leq u \leq t-1}[D(u, t)-S(u, t)-C,\right. \\
& D(s, t)-S(s, t)+C(s)-C]] \\
\leq & \sup _{0 \leq u \leq t}[D(u, t)-S(u, t)-C, D(0, t)-S(0, t)-C] \\
= & \sup _{0 \leq s \leq t}[D(s, t)-S(s, t)-C] \\
= & \sup _{0 \leq s \leq t}[D(s, t)-\alpha(t-s)+\alpha(t-s)+\beta(t-s) \\
& -\beta(t-s)-S(s, t)]-C \\
\leq & \sup _{0 \leq s \leq t}[D(s, t)-\alpha(t-s)]+\sup _{0 \leq s \leq t}[\beta(t-s)-S(s, t)] \\
& +\sup _{0 \leq s \leq t}[\alpha(s)-\beta(s)]-C .
\end{aligned}
$$

The right-hand side of (13) indicates a sufficient condition for $\mathbb{P}\{L P S(C, t)>0\}$. With $\mathbb{P}\left\{\sup _{0 \leq s \leq t}[D(s, t)-\alpha(t-s)]>\right.$ $x\} \leq \varepsilon_{d}(x), \mathbb{P}\left\{\sup _{0 \leq s \leq t}[\beta(t-s)-S(s, t)]>x\right\} \leq \varepsilon_{s}(x)$, the theorem is proved based on Lemma 1 .

\section{CASE STUDY}

\section{A. Description of the Data Set}

As a case study, we consider the Santa Catalina Island, which is 26 miles off the coast of Southern California, USA. It is with 76 square miles in area and has 54 miles of coastline. Currently, the electricity on Catalina is generated from a central diesel plant, and the island is served by three $12 \mathrm{kV}$ distribution circuits which are separate from the grid on the California mainland. It is desirable to reduce diesel-based generation for both environmental and economical reasons.
This paper aims to investigate the feasibility of replacing diesel generation with generation from renewable resources.

We use data profiles including power load, solar PV generation, and wind generation for analytical study. The hourly variation of data profiles for 31 days in January, 2010 are shown in Fig. 2, 4, and 6. They are obtained at geographical locations near Santa Catalina Island with similar meteorological characteristics:

- Energy generation profile: Based on the typical meteorological year (TMY) data sets derived from the National Solar Radiation Data Base (NSRDB) archives [2], the hourly per unit $\left(35 \mathrm{~m}^{2}\right)$ solar PV energy generation data for Long Beach, California, is calculated using the System Advisor Model [3]. The hourly energy generation data for a wind turbine located off an island near Santa Barbara, California is obtained from Western Wind Resources Data set from the National Renewable Energy Laboratory (NREL) [4].

- Load profile: The peak values for Santa Catalina Island are obtained by personal communication with researchers from Southern California Edison [5].

The cumulative per unit solar generation, per turbine wind generation and load profiles are depicted in Fig. 3, 5, and 7. With the typical power load and generation profiles for certain period, we address the design question: given the different renewable resource configurations, what are the appropriate amounts of battery storage capacity needed to ensure a certain level of power supply reliability?

\section{B. Performance Evaluations}

With the data given above, using curve fitting, we could get the bounding function with respect to the stochastic demand/supply curve according to Definitions 1 and 2. The demand/supply curve function is based on the measure of the long time mean rate. As the bounding function is non-negative and non-increasing function, here we use some convex exponential decay functions to fit the bounding functions.

For the energy demand process, we can get a stochastic demand curve $\alpha(t)$ with bounding function $\varepsilon_{d}$, denoted by $D \sim\left\langle\varepsilon_{d}, \alpha\right\rangle$. For the solar power supply, we assume all the PV panels are homogeneous. Based on the per unit data profile, given the total area of PV panels, $N_{p}$, we can find a stochastic supply curve $\beta^{p}(t)$, with bounding function $\varepsilon_{s}^{p}$, denoted by $S^{p} \sim\left\langle\varepsilon_{s}^{p}, \beta^{p}\right\rangle$. Similarly, all the wind turbines are also assumed to be homogeneous. Based on the per turbine data profile, given the number of wind turbines, $N_{w}$, we can find a stochastic supply curve $\beta^{w}(t)$ for the wind energy supply with bounding function $\varepsilon_{s}^{w}$, denoted by $S^{w} \sim\left\langle\varepsilon_{s}^{w}, \beta^{w}\right\rangle$. To aggregate heterogeneous power supply sources together, we then introduce the energy aggregation property:

Theorem 2: (Aggregation Property) Consider a power system that consists $N$ generators in parallel. If each power generator $(n=1,2, \ldots, N)$ provides a stochastic energy supply curve $S_{n} \sim\left\langle\varepsilon_{n}, \beta_{n}\right\rangle$, then the power system provides a stochastic supply curve $S \sim\langle\varepsilon, \beta\rangle$ with $\beta(t)=\beta_{1}(t)+\beta_{2}(t)+$ $\ldots+\beta_{N}(t), \varepsilon(x)=\varepsilon_{1} \otimes \varepsilon_{2} \otimes \ldots \otimes \varepsilon_{N}(x)$. 


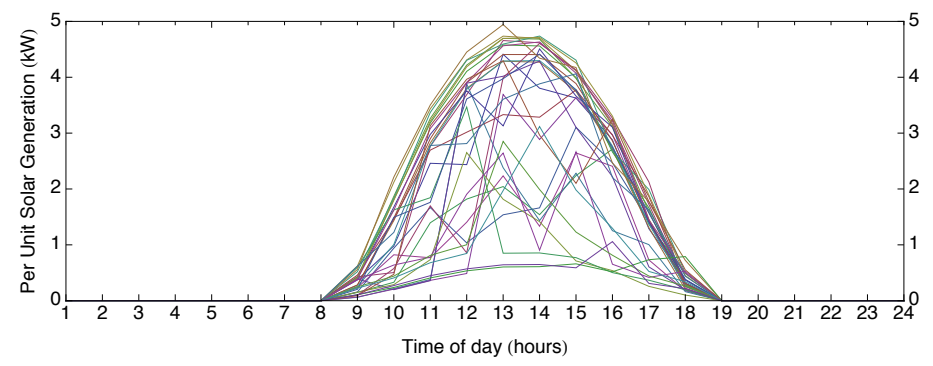

Fig. 2. Per unit solar generation profiles in Long Beach, CA

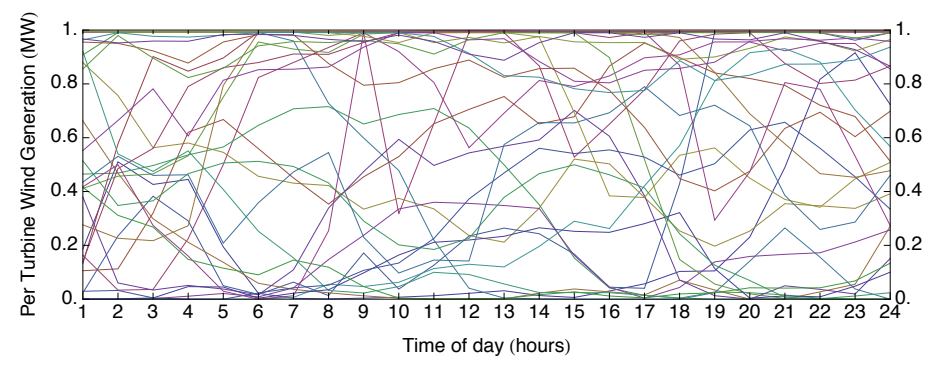

Fig. 4. Per turbine wind generation profiles on an island near Santa Barbara

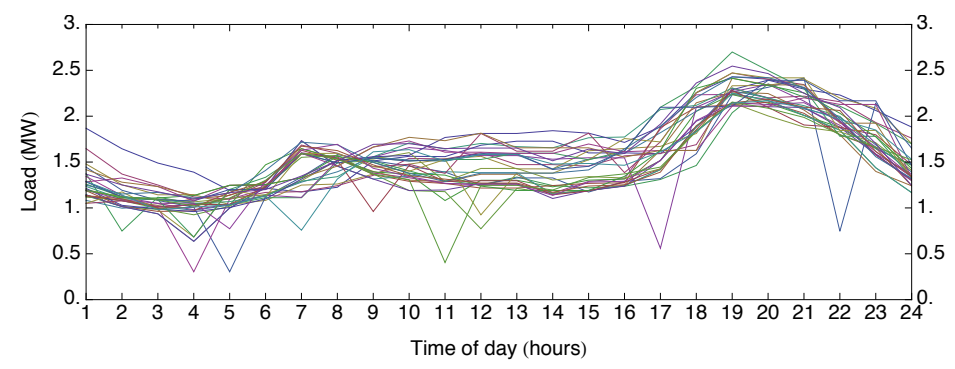

Fig. 6. Daily load profiles on Santa Catalina Island

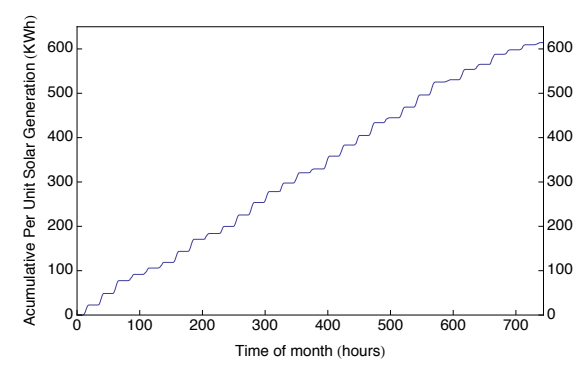

Fig. 3. Cumulative per unit solar generation profiles

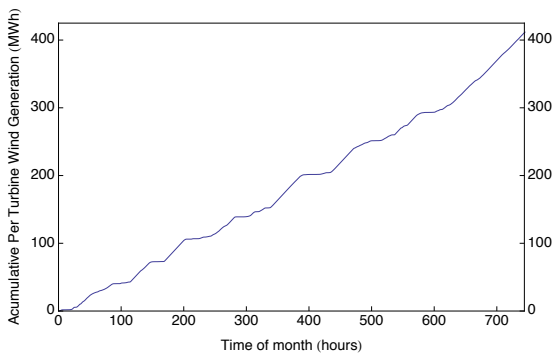

Fig. 5. Cumulative per turbine wind generation profiles

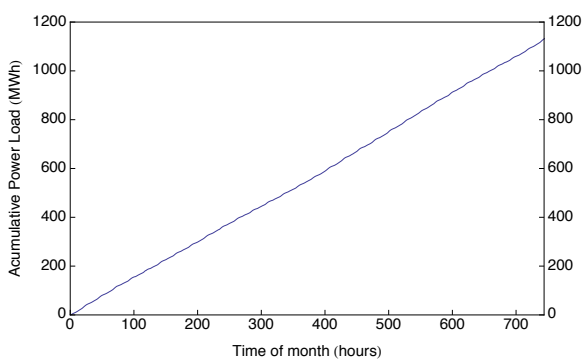

Fig. 7. Cumulative load profiles on Santa Catalina Island
Proof: Here we only illustrate the 2 generators case, from which the proof can be easily extended to the $N$ generators one. As $S(t)$ is the aggregation of 2 power supplies, we have $\forall 0 \leq s \leq t, S(s, t)=S_{1}(s, t)+S_{2}(s, t)$. For any function of $\beta_{n}(t), n=1,2$, we have

$$
\begin{aligned}
& \sup _{0 \leq s \leq t}\left[\left(\beta_{1}(t-s)+\beta_{2}(t-s)\right)-S(s, t)\right] \\
& \leq \sup _{0 \leq s \leq t}\left[\beta_{1}(t-s)-S_{1}(s, t)\right]+\sup _{0 \leq s \leq t}\left[\beta_{2}(t-s)-S_{2}(s, t)\right] .
\end{aligned}
$$

With the above assumptions, we have $\mathbb{P}\left\{\sup \left[\beta_{1}(t-s)-\right.\right.$ $\left.\left.S_{1}(s, t)\right]\right\} \leq \varepsilon_{1}(x)$, and $\mathbb{P}\left\{\sup _{0 \leq s \leq t}\left[\beta_{2}(t-s)-S_{2}(s, t)\right]\right\} \leq$ $\varepsilon_{2}(x)$, with Lemma 1, the theorem is proved.

\section{Independent Analysis}

In order to improve the bounding functions, we can further exploit the independence between different processes. In the rest of this paper, we use $[x]_{1}$ to denote the minimum of the given number $x$ and 1, i.e., $[x]_{1} \equiv \min \{x, 1\}$.

Lemma 2: For non-negative random variables $X$ and $Y$, with their complementary cumulative distribution functions
(CCDF) $F^{c}(x)$ and $G^{c}(x)$. If they are independent and $F^{c}(x) \leq \varepsilon_{f}(x)$ as well as $G^{c}(x) \leq \varepsilon_{g}(x)$, there holds

$$
\mathbb{P}\{X+Y>x\}=1-\mathbb{P}\{X+Y \leq x\} \leq 1-\varepsilon_{f}^{c} * \varepsilon_{g}^{c}(x),
$$

where the operator $*$ denotes the Stieltjes-Convolution, and $\varepsilon_{f}^{c}(x)=1-\left[\varepsilon_{f}(x)\right]_{1}$, and $\varepsilon_{g}^{c}(x)=1-\left[\varepsilon_{g}(x)\right]_{1}$.

The proof of the lemma is based on the StieltjesConvolution, which is given in [1], and it is omitted here due to page limitations. It can be verified that the bound in Lemma 2 is much better than the one calculated by Lemma 1 , and we have the following corollary for a better bound:

Corollary 1: Under the same condition as Theorem 2, if the energy supply process $S_{n},(n=1,2, \ldots, N)$ are stochastically independent, then the power system provides a stochastic supply curve $S \sim\langle\varepsilon, \beta\rangle$ with $\beta(t)=\beta_{1}(t)+\beta_{2}(t)+\ldots+\beta_{N}(t)$, $\varepsilon(x)=1-\varepsilon_{1}^{c} * \varepsilon_{2}^{c} * \ldots * \varepsilon_{N}^{c}(x)$, where $\varepsilon_{n}^{c}(x)=1-\left[\varepsilon_{n}(x)\right]_{n}$, $(n=1,2, \ldots, N)$.

If the energy demand and supply processes are independent, we can also improve the bounding function derived in Theorem 1 accordingly:

Corollary 2: With the same condition as Theorem 1, if the energy demand process and energy supply process are 


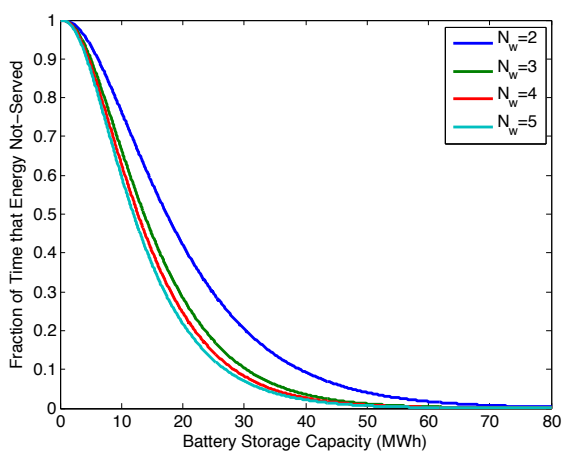

Fig. 8. The fraction of time that energy not-served as a function of battery storage capacity with $N_{p}=2 \times 10^{3}$ and different number of wind turbines

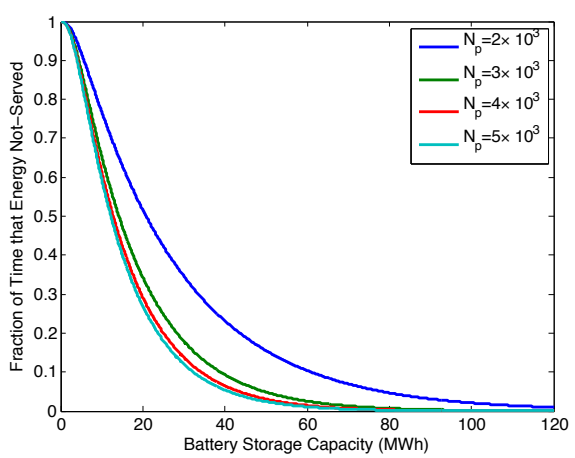

Fig. 9. The fraction of time that energy not-served as a function of battery storage capacity with $N_{w}=1$ and different number of PV units

stochastically independent, there holds

$$
\mathbb{P}\{L P S(C, t)>0\} \leq 1-\varepsilon_{d}^{c} * \varepsilon_{s}^{c}\left(C-\sup _{0 \leq s \leq t}[\alpha(s)-\beta(s)],\right.
$$

where $\varepsilon_{d}^{c}(x)=1-\left[\varepsilon_{d}(x)\right]_{1}$, and $\varepsilon_{s}^{c}(x)=1-\left[1, \varepsilon_{s}(x)\right]_{1}$.

\section{Numeric Results}

With different configurations of power generation, the aggregate energy supply from the solar and wind power can be calculated based on Corollary 2. For a given battery capacity $C$, the LPSP is provided by Corollary 1 . Together with (9), we can get the FTNS over the time period of the given data profile, i.e., $T=744$ in January.

Fig. 8 depicts the FTNS versus battery storage capacity with a fixed level of solar generation, i.e., $N_{p}=2 \times 10^{3}$, for different number of wind turbines, $N_{w}$. As $N_{w}$ increases, the FTNS decreases with the same battery capacity and approaches a constant value. For a targeting FTNS value of 0.01 , we can get the battery capacity requirement with different energy configuration, i.e., when the wind turbines increases from 2 units to 5 with other setting fixed, the battery capacity requirement reduces from $66.5 \mathrm{MWh}$ to $51.3 \mathrm{MWh}, 48.4$ $M W h$ and 46.6 $M W h$ respectively.

Fig. 9 shows the FTNS versus battery capacity for different levels of solar generation with a single wind turbine. We notice that FTNS decreases as the battery capacity increases. Similar to the trend with increasing $N_{w}$ in Fig.8, as $N_{p}$ increases, the transition from high FTNS to low FTNS sharpens with the increasing of battery capacity. Given a targeting FTNS value, further increasing the wind turbines would have less impact in reducing the battery requirement due to the fluctuating nature of the renewable power sources.

\section{CONClusion}

In this paper, we discuss how the concepts of stochastic network calculus is applicable to the design of a power grid. Motivated by the challenges associated with integration of renewable energy resources in to the electric grid due to power intermittency, we introduce a new approach to asses the system reliability based on stochastic network calculus. By adjusting the framework of stochastic network calculus, we build a stochastic model to analyze the achievable level of system reliability with appropriate number of PV cells, wind turbines, and energy storage capacity. We also consider a case study on Santa Catalina Island, and investigate the feasibility of replacing diesel generation entirely with PV panels and wind turbines, supplemented with energy storage. An important issue of stochastic network calculus is to find proper supply and demand models, and we would further investigate alternative models to better suit the analysis.

\section{ACKNOWLEDGMENT}

This research is supported by the State Scholarship Fund of China, and the National Natural Scientific Foundation of China (No. 61020106002 and No.60973107). The authors wish to thank Florin Ciucu from Deutsche Telekom Labs for fruitful discussions, and Rui Huang from Caltech for interaction in generating the data sets.

\section{REFERENCES}

[1] Yuming Jiang, Yong Liu. Stochastic Network Calculus. Springer, 2008.

[2] http://rredc.nrel.gov/solar/old_data/nsrdb/1991-2005/tmy3/

[3] https://www.nrel.gov/analysis/sam/

[4] http://wind.nrel.gov/Web_nrel/

[5] http://www.sce.com/AboutSCE/Regulatory/loadprofiles/default.htm

[6] F. Ciucu, A. Burchard, and J. Liebeherr. A network service curve approach for the stochastic analysis of networks. ACM SIGMETRICS Performance Evaluation Review, 33(1):279-290, June, 2005.

[7] M. Fidler. An end-to-end probabilistic network calculus with moment generating functions. In Proceedings of IWQoS, 2006.

[8] J.-Y. Le Boudec and P. Thiran. Network Calculus: A Theory of Deterministic Queueing Systems for the Internet. Springer-Verlag, 2001.

[9] Borowy BS, Salameh ZM. Methodology for optimally sizing the combination of a battery bank and PV array in a wind/PV hybrid system. IEEE Transactions on Energy Conversion 1996;

[10] Tina G, Gagliano S, Raiti S. Hybrid solar/wind power system probabilistic modeling for long-term performance assessment. Solar Energy 2006;80(5):578-588.

[11] Yang HX, Zhou W, Lu L, Fang ZH. Optimal sizing method for standalone hybrid solarewind system with LPSP technology by using genetic algorithm. Solar Energy 2008;82(4):354-367.

[12] Zhou W, Lou C, Li Z, Lu L, Yang H. Current status of research on optimum sizing of stand-alone hybrid solarewind power generation systems. Applied Energy 2010;87(2):380-9.

[13] http://reviews.cnet.com/8301-13746_7-20016664-48.html

[14] H. Xu, U. Topcu, S. H. Low, C. Clarke and M. Chandy. Load-shedding probabilities of power systems with renewable power generation and energy storage. in Allerton Conference on Communication, Control and Computing, Monticello, IL, September/October 2010.

[15] Yang HX, Lu L, Zhou W. A novel optimization sizing model for hybrid solarwind power generation system. Solar energy 2007;81(1):7684. 